\title{
La composition du lait de vache de la région de Plovdiv en Bulgarie et de Ioannina en Grèce
}

\author{
par \\ B. VEINOGLOU*, M. BALTADJIEVA**, E. ANIFANTAKIS*** \\ et M. EDGARYAN****
}

\section{INTRODUCTION}

Cette étude a été réalisée dans le cadre de la coopération du laboratoire de Technologie Laitière de l'Institut de Technologie Alimentaire de Plovdiv et du laboratoire homologue de l'Ecole Supérieure d'Agriculture d'Athènes.

Elle concerne la composition du lait de vache produit dans la région de Plovdiv en Bulgarie et dans celle de Ioannina en Grèce. Nous avons choisi ces deux régions à cause de leur production importante en lait de vache et aussi parce que les deux laboratoires se sont procurés les quantités nécessaires à la préparation de fromages expérimentaux. Il faut noter en plus qu'il manque une pareille étude sur la composition de lait de vache des régions mentionnées.

\section{MATERIELS ET METHODES}

\section{Origine des échantillons}

Des échantillons de lait de vache, représentatifs de la quantité produite dans les deux régions, ont été prélevés tous les 2 jours durant

\footnotetext{
* Professeur à la chaire de Technologie Laitière à l'Ecole Supérieure d'Agriculture d'Athènes.

** Vice-Recteur de l'Institut de Technologie Alimentaire de Plovdiv (Bulgarie).

*** Maître de conférences à la chaire de Technologie Laitière à l'Ecole Supérieure d'Agriculture d'Athènes.

**** Assistant à la chaire de Technologie Laitière de l'Institut de Technologie Alimentaire de Plovdiv (Bulgarie).
} 
la lactation des années 1979 et 1980. Au total nous avons examiné 72 échantillons de chaque région.

Les analyses chimiques ont été effectuées dans les deux laboratoires.

\section{Méthodes analytiques}

Les analyses de protéines totales et de caséine ont été effectuées selon les normes 20-1962 et 29-1964 de la Fédération Internationale de Laiterie ; le lactose et la matière sèche selon les normes 28A-1974 et 21-1962. L'analyse de la teneur en cendres et en calcium a été effectuée selon le "British Standard " 1741/1963.

La matière grasse a été déterminée selon la méthode Gerber, l'acidité avec la méthode Dornic, le poids spécifique à $15^{\circ} \mathrm{C}$ avec le lactodensimètre.

\section{RESULTATS ET DISCUSSION}

La composition moyenne des laits de vache des deux pays, d'après les analyses que nous avons effectuées durant les lactations des années 1979 et 1981, est présentée au tableau 1.

L'étude statistique des données de nos analyses nous permet de constater :

1. Le lait produit dans la région de Plovdiv est considérablement plus riche que celui de Ioannina, en matière sèche, protéine totale, caséine et calcium, mais plus pauvre en cendres. Les deux laits ont une différence significative en protéines solubles et en lactose. Les différences que nous avons constatées sont dues principalement aux races élevées. En dehors des races, l'alimentation et les conditions climatiques sont les facteurs les plus importants de ces différences.

2. Les coefficients de variations ne montrent pas une tendance homogène. Pour certains constituants ils sont supérieurs pour le lait de Ioannina et pour d'autres pour le lait de Plovdiv. Il faut signaler que dans les deux cas nous avons des variations en teneur en protéines plus importantes que pour les autres constituants, contrairement à ce que nous attendions.

3. La matière sèche dégraissée du lait de Ioannina est inférieure à celle imposée par la réglementation laitière grecque [8].

4. La teneur en calcium du lait se trouve dans les limites mentionnées dans la bibliographie, quoique le lait de Ioannina approche des limites inférieures [5]. Une des raisons de cette différence, doit être la basse teneur en caséine de lait de Ioannina.

Les résultats du tableau 1 présentent une image générale de la composition du lait de deux régions, mais sans présenter les variations 
TABLEAU 1

Composition moyenne du lait de vache de Ioannina et de Plovdiv durant la lactation des années 1979 et 1980

\begin{tabular}{|c|c|c|c|c|c|c|c|c|c|c|c|}
\hline & \multicolumn{5}{|c|}{ Ioannina } & \multicolumn{5}{|c|}{ Plovdiv } & \multirow[b]{2}{*}{ t-test } \\
\hline & Moyenne & $\begin{array}{c}\text { Ec. } \\
\text { typique }\end{array}$ & $\begin{array}{c}\text { Coeffi- } \\
\text { cient de } \\
\text { variation }\end{array}$ & Max. & Min. & Moyenne & $\begin{array}{c}\text { Ec. } \\
\text { typique }\end{array}$ & $\mid \begin{array}{c}\text { Coeffi- } \\
\text { cient de } \\
\text { variation }\end{array}$ & Max. & Min. & \\
\hline Extrait sec p. 100 & 12,02 & 0,282 & 2,35 & 12,56 & 11,54 & 12,96 & 0,434 & 3,35 & 14,17 & 12,36 & 8,93 \\
\hline Matière sèche p. 100 & 3,64 & 0,134 & 3,69 & 3,92 & 3,39 & 3,94 & 0,129 & 3,28 & 4,15 & 3,70 & 7,68 \\
\hline Protéines totales p. 100 & 3,18 & 0,139 & 4,38 & 3,44 & 2,93 & 3,54 & 0,219 & 6,20 & 3,95 & 3,19 & 6,65 \\
\hline Caséine p. 100 & 2,36 & 0,123 & 5,19 & 2,58 & 2,15 & 2,67 & 0,219 & 8,20 & 3,09 & 2,18 & 5,85 \\
\hline Protéine soluble p. 100 & 0,82 & 0,050 & 5,99 & 0,94 & 0,73 & 0,87 & 0,095 & 10,86 & 1,04 & 0,70 & 1,63 \\
\hline Lactose p. 100 & 4,51 & 0,136 & 3,02 & 4,77 & 4,26 & 4,60 & 0,148 & 3,22 & 4,80 & 4,44 & 2,03 \\
\hline Cendre p. 100 & 0,758 & 0,027 & 3,56 & 0,819 & 0,700 & 0,729 & 0,026 & 3,51 & 0,778 & 0,695 & 3,84 \\
\hline Calcium p. 100 & 0,107 & 0,006 & 5,70 & 0,116 & 0,095 & 0,124 & 0,004 & 2,91 & 0,131 & 0,117 & 11,44 \\
\hline Densité p. 100 & 1,0306 & - & - & 1,0315 & 1,0299 & 1,0298 & - & - & 1,0312 & 1,027 & - \\
\hline Acidité p. 100 & 0,16 & - & - & 0,17 & 0,16 & 0,16 & - & - & 0,18 & 1,4 & - \\
\hline $\begin{array}{l}\text { pH } \\
\text { M.S. dégraissée }\end{array}$ & 6,60 & - & - & 6,65 & 6,59 & 6,57 & - & - & 6,62 & 6,45 & - \\
\hline
\end{tabular}


de chacun des constituants durant la lactation. Ces variations sont présentées dans les figures 1 à 7 , pour l'extrait sec, la matière grasse, les protéines totales, la caséine, le lactose, les cendres et le calcium.

5. En remarquant que la plus grande teneur du lait de Ioannina en cendres, en relation avec celle du lactose et du calcium, nous pouvons penser que les vaches de cette région souffrent en plus de mammites, point qu'il faut examiner.

6. Au point de vue valeur fromagère, le lait de la région de Plovdiv est meilleur que celui de Ioannina, car il est plus riche en matière grasse, protéines et calcium, d'où une différence pour le rendement en fromage de 10 p. 100 en faveur du lait de Plovdiv.

Dans les figures 1 à 4 nous constatons que pour le lait de la région de Ioannina, la matière grasse, les protéines totales, la caséine et l'extrait sec montrent une même variation pendant la lactation : les valeurs supérieures sont au début et à la fin de l'année et les plus basses aux mois de juin, juillet et août.

Les variations saisonnières de la composition du lait sont semblables à celles constatées par Loganathan et THOMPSON [7] et OvERMAN et al. [9]. Il est caractéristique que la courbe de la production moyenne de la région est inversée comme nous voyons dans la figure 8. Dans le cas du lait de Plovdiv nous avons les mêmes variations pour la matière grasse et l'extrait $\mathrm{sec}$, mais au contraire nous n'avons pas la même variation pour les protéines.

Les cendres du lait montrent une variation pendant l'année, (fig. 6), sans avoir des tendances saisonnières. Les résultats sont similaires pour le calcium (fig. 7).

Si nous examinons les données analytiques des 2 années, par région, nous pouvons remarquer une différence de la composition de 1 année à l'autre, effet que nous pouvons attribuer aux conditions climatologiques qui ont prédominé. II est évident que dans le cas de deux régions le lait de 1979 était plus riche que celui de 1980. Les différences sont plus intéressantes pour le lait de Plovdiv, particulièrement du mois de mars au mois d'octobre, comme nous pouvons le constater dans les fig. 9 et 10 , que nous proposons comme exemple et qui concernent l'extrait sec du lait.

Nous avons aussi examiné s'il existe une corrélation quantitative entre les différents constituants du lait.

Dans ce cadre nous avons calculé les coefficients de variation ( $r$ ) entre la matière grasse et l'extrait $\mathrm{sec}$, les protéines totales et l'extrait sec dégraissé, les protéines et la caséine et nous avons trouvé $+0,987$, $+0,871,+0,837$ et $+0,907$ respectivement pour le lait de Ioannina. Pour le lait de Plovdiv les valeurs sont $+0,580,+0,659,+0,348$ et $+0,904$. Nous constatons qu'il existe une corrélation positive dans tous les cas mais pour le lait de Plovdiv les coefficients sont plus petits. 


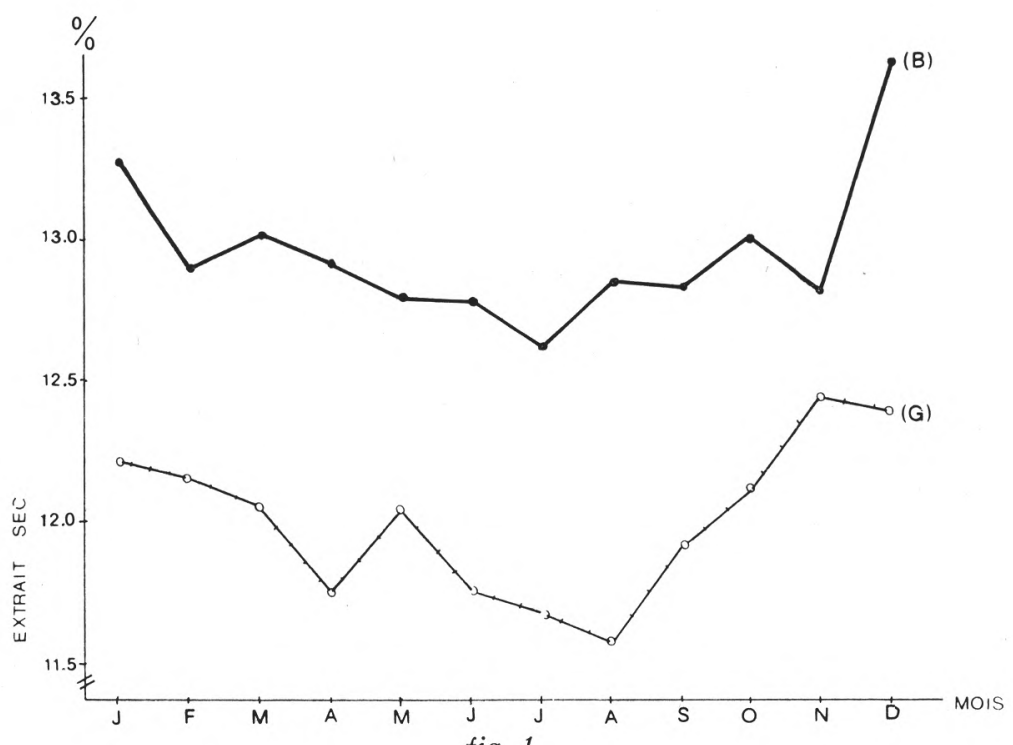

fig. 1

Variation saisonnière en extrait sec du lait de vache de Ioannina $(G)$ et Plovdiv (B).

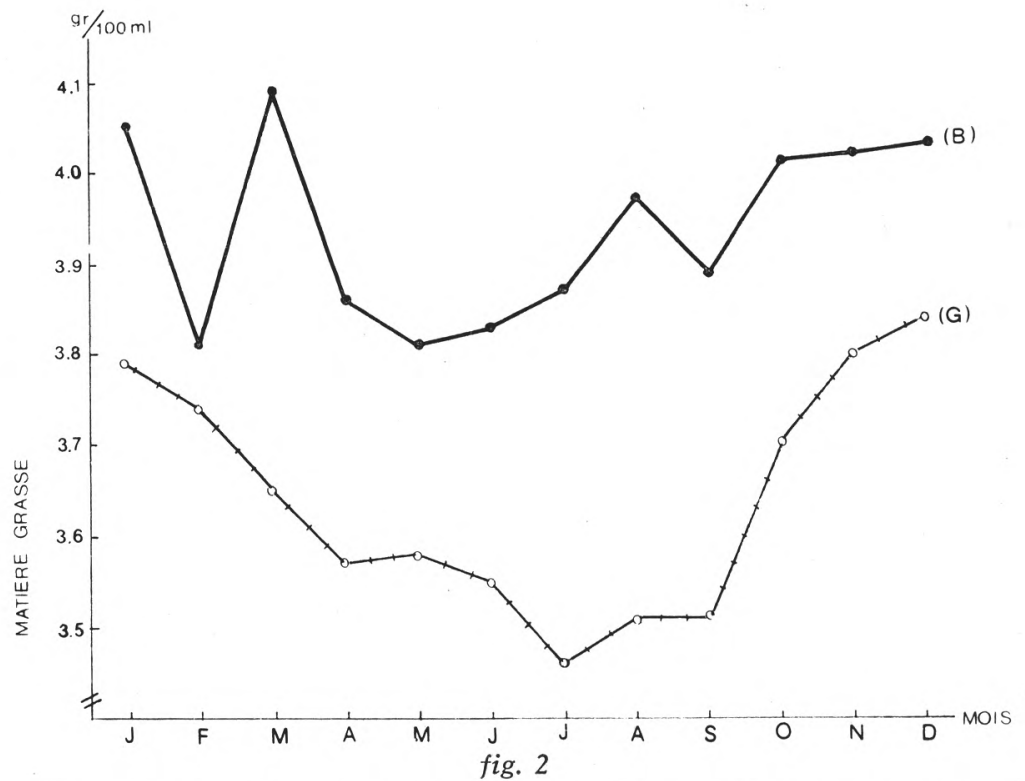

Variation saisonnière en matière grasse du lait de vache de Ioannina (G.) et Plovdiv (B). 


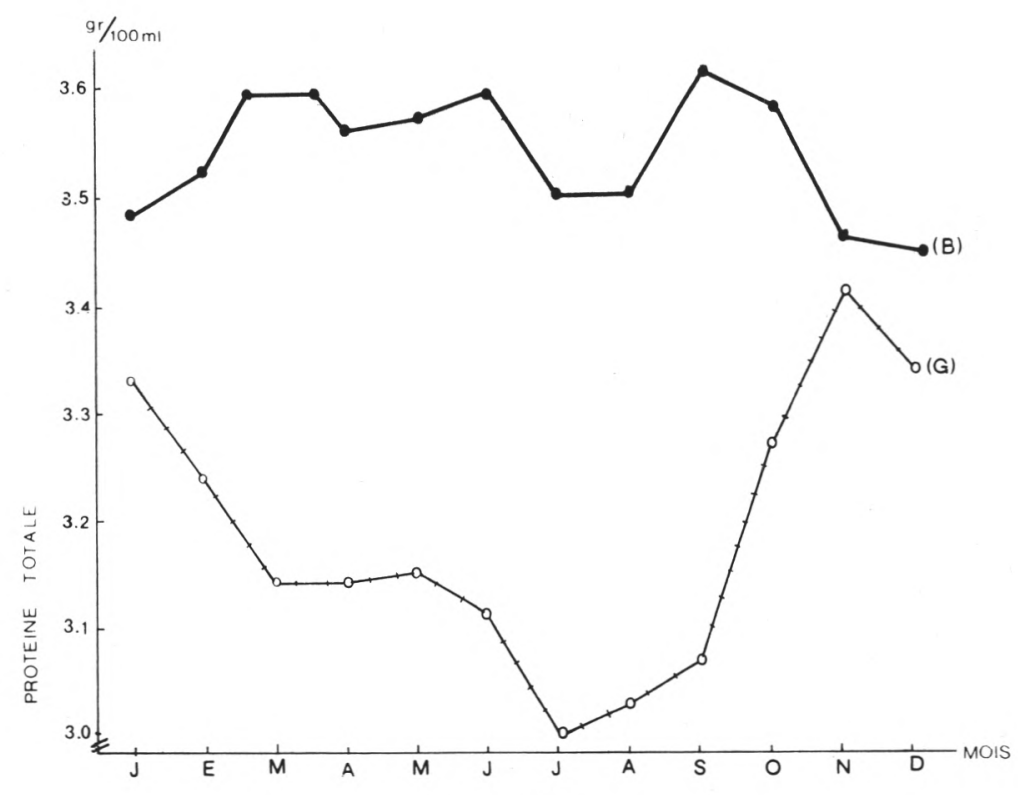

fig. 3

Variation saisonnière en protéine totale du lait de vache de Ioannina (G) et Plovdiv (B).

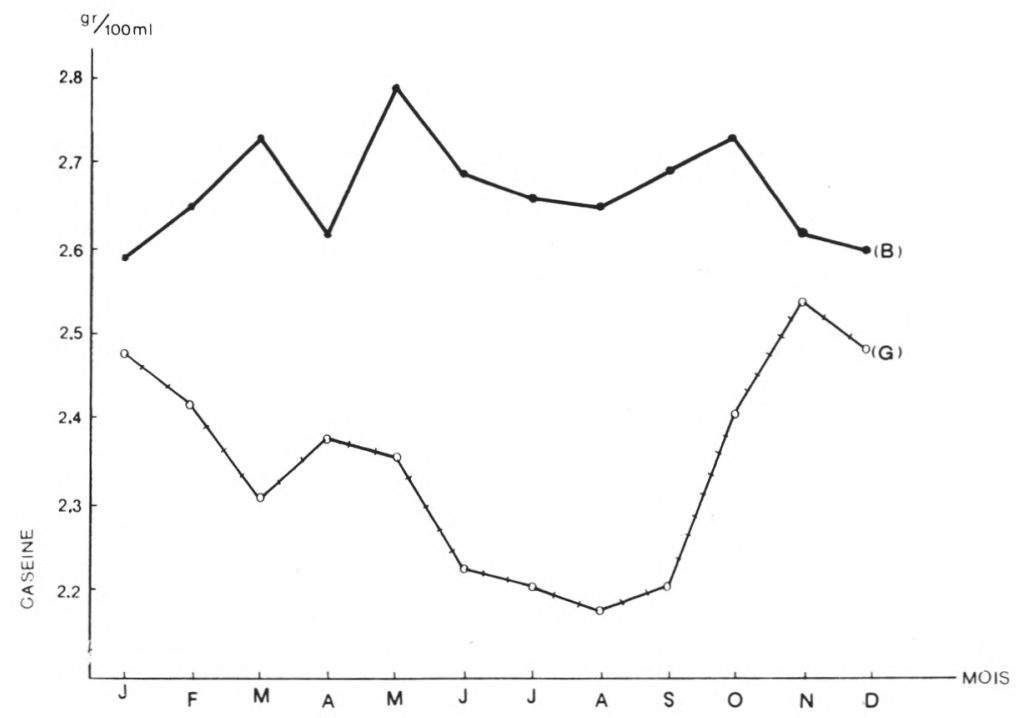

fig. 4

Variation saisonnière en caséine du lait de vache de Ioannina (G) et Plovdiv (B). 


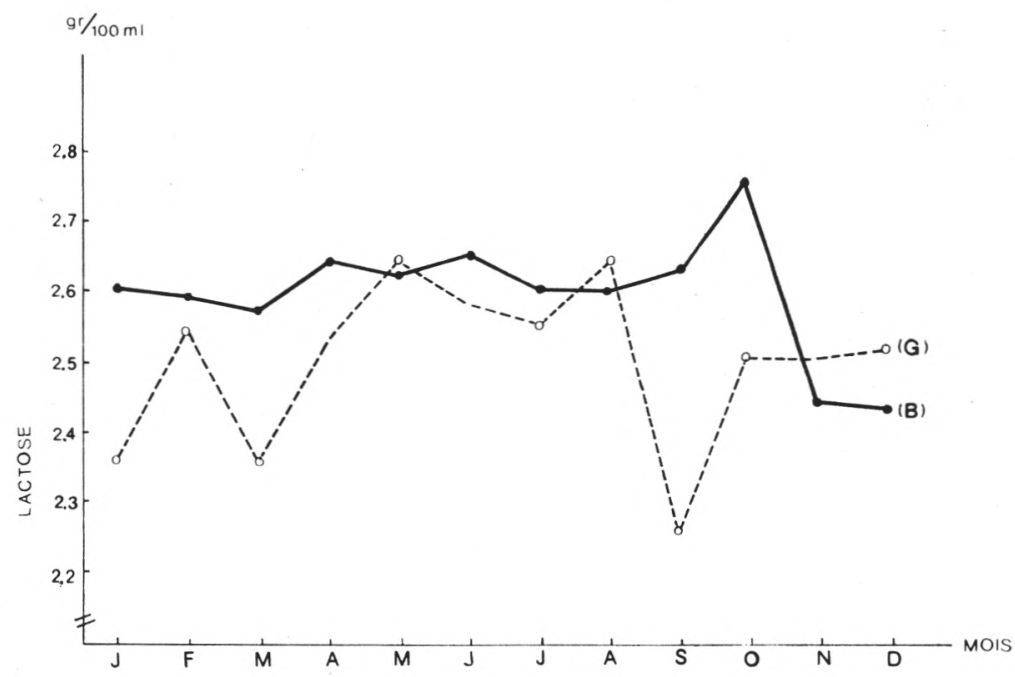

fig. 5

Variation saisonnière en lactose du lait de vache de Ioannina (G) et Plovdiv (B).

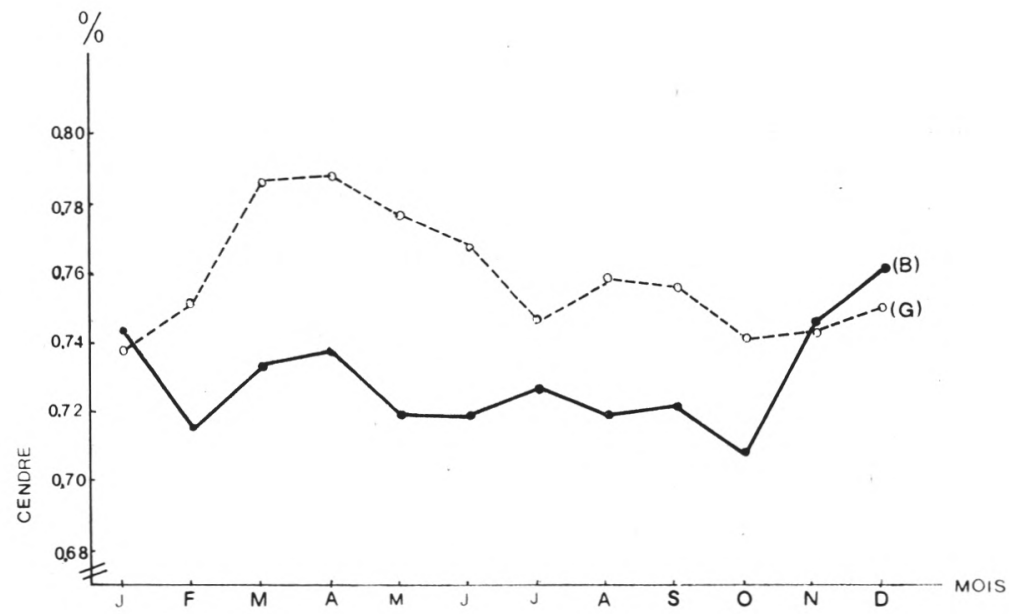

fig. 6

Variation saisonnière en cendre du lait de vache de Ioannina (G) et Plovdiv (B). 


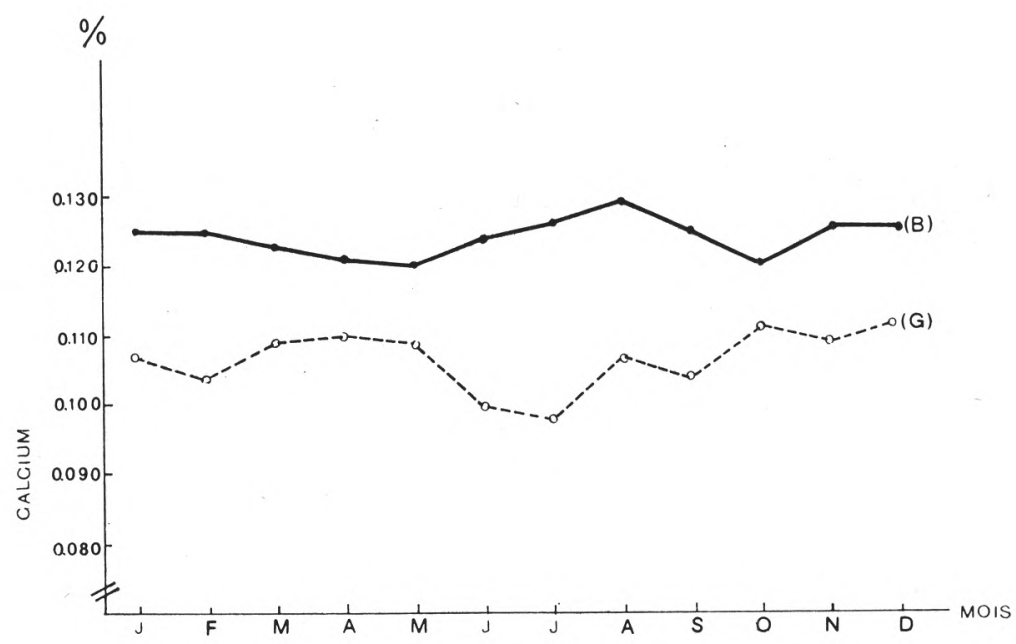

fig. 7

Variation saisonnière en calcium du lait de vache de Ioannina (G) et Plovdiv (B).

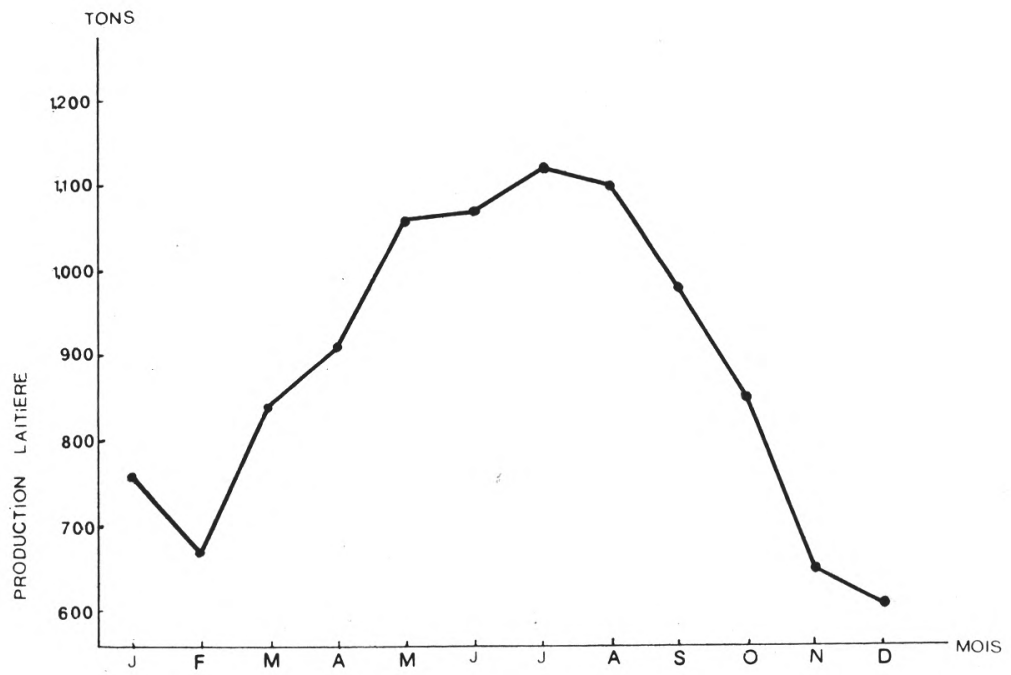

fig. 8

Variation de la production moyenne mensuelle du lait de vache de la région de Ioannina pendant les années 1979 et 1980. 


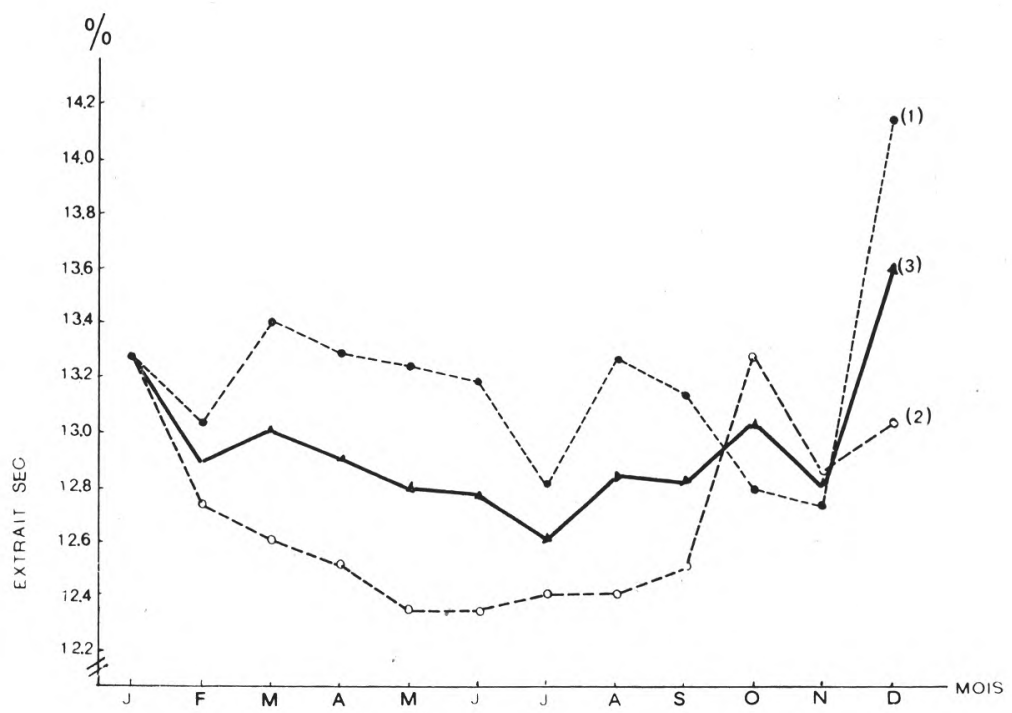

fig. 9

Variation saisonnière en extrait sec du lait de la région de Plovdiv pendant les années 1979 (1) et 1980 (2) et la moyenne de 2 ans (3).

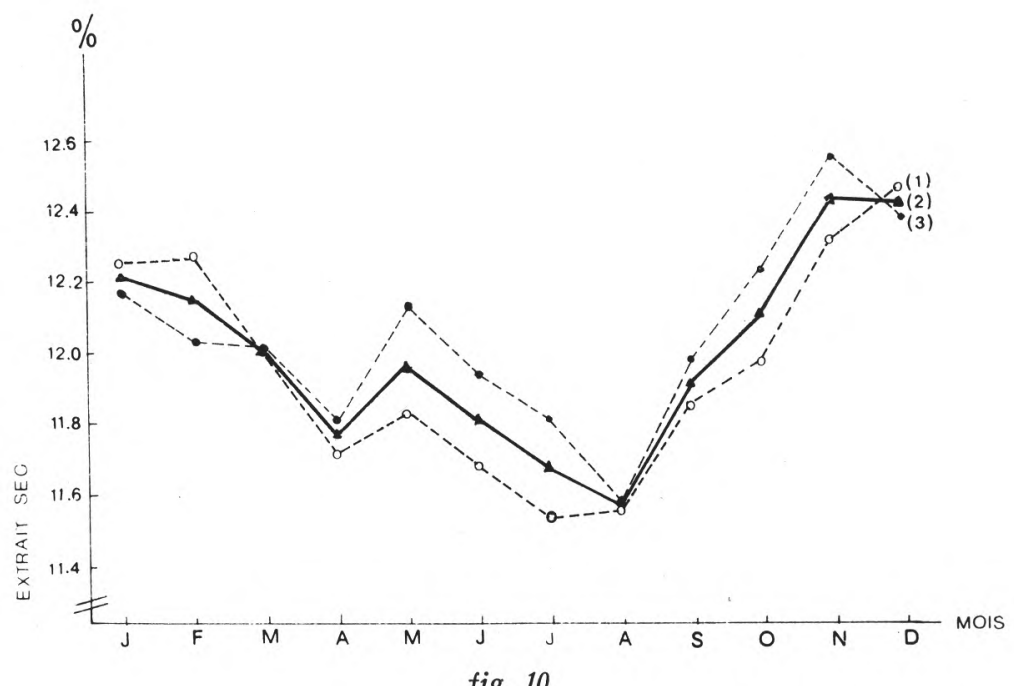

fig. 10

Variation saisonnière en extrait sec du lait de la région de Ioannina pendant les années 1979 (1) et 1980 (2) et la moyenne de 2 ans (3). 
TABLEAU 2

Composition du lait de vache de différents pays

\begin{tabular}{|c|c|c|c|c|c|c|}
\hline Pays & Ex. sec p. 100 & M.G. p. 100 & Protéine & Lactose p. 100 & Cendre p. 100 & Référence \\
\hline Australie & 12,4 & 3,8 & - & - & - & [11] \\
\hline Bulgarie & 11,63 & 3,15 . & 3,34 & - & 0,68 & [3] \\
\hline Finlande & 13,05 & 4,32 & 3,34 & 4,76 & - & {$[10]$} \\
\hline France & - & 3,71 & 3,24 & - & - & [2] \\
\hline Japon & 11,53 & 3,42 & 2,87 & 4,54 & 0,70 & [12] \\
\hline Pologne & 12,68 & 3,90 & 3,29 & - & - & [4] \\
\hline Russie & 12,42 & 3,70 & 3,29 & 4,25 & 0,72 & [1] \\
\hline Suède & 12,65 & 3,92 & 3,15 & 4,92 & - & {$[6]$} \\
\hline
\end{tabular}


En comparant les résultats de notre recherche aux données que l'on peut trouver dans la bibliographie nous constatons que le lait de la région de Plovdiv est un des plus riches en extrait sec, au contraire le lait de Ioannina est pauvre (tab. 2).

En même temps nous avons examiné certaines caractéristiques physicochimique comme la densité, $\mathrm{pH}$ et l'acidité et nous avons constaté qu'il n'existe pas une différence significative entre le lait de deux régions.

\section{Rés u mé}

La composition du lait de vache des régions de Plovdiv en Bulgarie et de Ioannina en Grèce a été examinée durant les lactations des années 1979 et 1980 .

La composition moyenne, après avoir analysé 72 échantillons de lait grec et 72 échantillons de lait bulgare, est, respectivement : Extrait sec 12,02-12,96 p. 100, matière grasse 3,64-3,94 p. 100, protéines totales $3,18-3,54$ p. 100 , caséine $2,36-2,67$ p. 100 , protéines solubles 0,82 0,87 p. 100 , lactose $4,51-4,60$ p. 100 , cendres $0,76-0,73$ p. 100 et calcium $0,107-0,124$ p. 100. D'après les données analytiques et leurs études statistiques il existe des différences importantes dans la composition du lait des deux régions. Celui de la région de Plovdiv est plus riche en extrait sec, matière grasse, protéines totales, caséine, calcium et plus pauvre en cendres.

La composition du lait varie sensiblement durant l'année et d'une année à l'autre. Pour le lait de Ioannina nous avons constaté que les teneurs de l'extrait sec, de la matière grasse, et des protéines, diminuent pendant les mois d'été et augmentent au début et à la fin de l'année. Pareil phénomène a été constaté pour l'extrait sec et la matière grasse. Les protéines montrent une variation importante.

\section{S u m m a ry}

THE COMPOSITION OF COW'S MILK PRODUCED IN PLOVDIV REGION IN BULGARIA AND IN THE IOANNINA REGION IN THE NORTH WEST GREECE

Cow's milk samples were taken during the years 1979 and 1980 at 10 days intervals from the regions of Plovdiv (Bulgaria) and Ioannina (Greece) and their chemical composition was studied. From the statistical analyses of the data the following conclusions were derived:

a) There are significant differences in the composition of the milk taken from the two regions. That of the Plovdiv region was richer in dry matter, fat, total proteins, casein and calcium and poorer in ash. There were non significant differences in their lactose and soluble nitrogen content. 
b) The composition of the milk of the two regions, shows significant differences during the year and from year to year. In the case of the milk from Ioannina it was found that, the dry matter, the fat and the proteins were decreasing during the summer and increasing towards the begining and the end of the year. Similar results were also obtained for the milk of the Plovdiv region for the dry matter and the fat.

c) There were not differences in $\mathrm{pH}$, acidity and specific gravity of the milk of the two regions.

d) The composition of the milk of the region of Ioannina and Plovdiv was found to be the following, respectively: Dry mater p. 100 12.02-12.96, fat p. $1003.64-3.94$, total proteins p. $1003.18-3.54$, soluble proteins p. $100 \quad 0.82-0.87$, lactose p. $1004.51-4.60$, ash p. $100 \quad 0.76-0.73$ and calcium p. 100 0.107-0.124.

Reçu pour publication en août 1981.

\section{Remerciements}

Nous remercions vivement $\mathrm{M}$. S. Kaminaridis pour la précieuse assistance qu'il nous a prodiguée lors de la réalisation de cette étude.

\section{Bibliographie}

[1] Abramova (O.) (1965). - Composition and quality of milk received at a dairy in Rostov, Dairy Sci. Abs., 28, 262.

[2] Grappin (R.), Ricordeau (G.), Mocouot (G.), Jeunet (R.) et Tassencourt (L.) (1967). - Variations saisonnières et annuelles de la richesse en matière grasse et en protéines de laits de producteurs appartenant à cinq régions de France. Revue Laitière française, 240, 71-82.

[3] Grigorov (K. H.) (1968). - Biochemical and Technological characteristics of cow's milk. I. Milk of Bulgarian Brown cattle. Vet. Med. Nauki Sof,, $5,59-65$.

[4] Jasiorowski (H.) and PoczynaJlo (S.) (1966). - Int. Dairy Congress, Section $\mathrm{A} / 21$.

[5] Jennes (R.) and Patton (S.) (1976). - In principles of dairy chemistry (book), 161.

[6] Ljunggren (B.) (1967), - Composition of incoming milk within the Central Dairy's province. Dairy Sci. Abs., 29, 2463.

[7] Loganathan (S.) and Thompson (N. R.) (1967). - Lactational trends of milk constituents. J. Dairy Sci., 50, 1009.

[8] Ministère des Affaires Financières (1972). - Code alimen.

[9] Overman (O. R.), Garett (O. F.), Wright (K. E.) and Sanmann (F. P.) (1939), Composition of Milk of Brown Swiss Cows. III. Agr. Expe. St. bull. 457.

[10] Peltola (E.), Antila (P.) and Malki (Y.) (1963). - Studies on the composition of Finnish cow's milk. Meijerit Aikakausk, 24, 3-18.

[11] TUCKeR (V.C.) (1968). - Composition of milk on the Atherton Tableland. Aust. J. Dairy Technology, 23, 36-47.

[12] Yamamoto (T.), Hamada (H.), Takahashi (K.), Chikuma (G.) and Koishcikawa (T.) (1967). - Investigation of the composition of milk in various districts of Japan. Bull. Natn. Inst. Anim. Ind., Chiba, 14, 11-22. 